\title{
NOTE PRÉLIMINAIRE \\ SUR LA PRÉSENGE DE SUBSTANCES ANTIBIOTIQUES CHEZ QUELQUES ESSENCES FORESTIÈRES
}

\author{
Marie-France MICHEL \\ Station de Recherches sur la Forêt et l'Environnement, \\ Centre de Recherches forestières d'Orléans, I. N.R. A., \\ Ardon, 45160 Olivet
}

\section{RÉSUMÉ}

Dans cette note sont rapportées les premières expériences mettant en évidence l'activité antimicrobienne du feuillage de plusieurs essences forestières, feuillus ou résineux.

En laboratoire, plusieurs tests microbiologiques ont été utilisés : antibiogrammes et étude des phytoncides émis par des feuilles broyées.

Plusieurs facteurs ont été analysés : spécificité à l'égard des souches bactériennes, variation de l'activité phytoncide en fonction des différentes essences testées d'une part, et des méthodes employées d'autre part.

C'est en ig29 que Tokis désigne sous le terme de phytoncides les substances antimicrobiennes produites ou émises par les végétaux supérieurs. Depuis, de nombreuses recherches ont été effectuées sur ce sujet et il semble que la plupart des espèces végétales produisent des substances antibiotiques inhibitrices de microorganismes, voire même d'autres êtres vivants, y compris des végétaux chlorophylliens (Michei, I975).

Longtemps, la notion d'antibiose, après avoir été créée par VuilLEMIN en I 889 pour caractériser 1'antagonisme s'exerçant par voie chimique entre les êtres vivants, a été restreinte aux interactions existant entre micro-organismes et le terme antibiotique désignait les substances chimiques inhibitrices produites par ces microorganismes (Eubactériales, Actinomycètes, Fungi imperfecti). Récemment, la définition du mot antibiotique a repris son sens étymologique large et s'applique à toute substance produite par des organismes vivants et inhibant à faible concen- 
tration la croissance d'autres organismes. Nous désignerons sous le terme de phytoncides les antibiotiques volatils émis par les végétaux supérieurs.

Plusieurs auteurs ont mis en évidence la présence de substances inhibitrices de la croissance de micro-organismes chez quelques feuillus et résineux. Différents organes ont été étudiés, tels que les feuilles (SMIrNofr, I967, I968, I972 ; PeiterKOVA et PETERKA, I972), l'écorce des trones ou des racines (KL.OPPING et VAN DER Kerr, I95I ; Hubbes, I966; Hubbes et Jung, I973; Jung, I965).

Dans cette étude préliminaire, nous avons étudié l'activité phytoncide des feuilles de plusieurs essences de feuillus et de résineux. Cette première étape dans l'étude de l'activité antibiotique des arbres a eu pour buts essentiels :

I. la mise en évidence des phénomènes d'inhibition in vitro ce qui impliquait la recherche exploratoire de matériels tests au point de vue, d'une part, des souches bactériennes et, d'autre part, d'essences ligneuses à fort pouvoir antibiotique et devant être utilisées ultérieurement comme essence de référence dans les études en enceinte d'exposition et in situ ;

2. 1a détermination des limites et des avantages des tests microbiologiques utilisés dans l'étude des antibiotiques employés en thérapeutique ;

3. la mise au point d'une méthode d'étude afin de tester l'efficacité des antibiotiques volatils émis par le feuillage des arbres.

\section{I. - MATÉRIEL E'T TECHNIQUES EXPÉRIMENTALES}

\section{I. - Choix et provenance des essences forestières}

Les différents échantillons de feuilles ou d'aiguilles testées pour leur activité phytoncide ont été prélevés à l'Arboretum d’Amance sur plusieurs espèces de résineux ou de feuillus. Il s'agit de Chamaecyparis nutkaensis spach (placeau 23), Thuya plicata D. Dox (placeau 24), Abies alba mill (placeau 5, Aude, France), Cedrus atlantica manetri (placeau 9), Pinus silvestris L. (placeau I3, Pologne), Larix decidua mill (placeau 5, Puy Saint-Vincent, Hautes-Alpes, France), Populus trichocarpa TORR et GRAY (placeau 23) et Sorbus aucuparia L. (placeau 4). Parmi ces essences, deux d'entre elles produisent des antibiotiques connus pour leurs propriétés antifongiques et antibactériennes : la nootkatine extraite du bois de coeur du C. nutkacnsis (AvlıxERdtMax, I950) et les thujaplicines $\alpha, \beta$ et $\gamma$ extraites de $T$. plicata (ERDTMAx et GRIPENBERG, I948).

$$
\text { I. 2. Souches bactériennes utilisées }
$$

Les souches bactériennes utilisées a fin de mettre en évidence l'activité inhibitrice des arbres forestiers proviennent de l'Institut Pasteur. Trois d'entre elles sont Gram ${ }^{+}:$Bacillus cereus, Bacillus thuringiensis VAR. thuringiensis, Staphylococcus aureus 209 P, ATCC 6538 P (souche employée comme souche de référence dans les études de tests de sensibilité aux antibiotiques) : la dernière, Eschevichia coli est Gram-

$$
\text { I. 3. - Préparation des extraits }
$$

Après prélèvement, les feuilles ou les aiguilles sont rapidement broyées au broycur (WaringBlendor) et sont mises à macérer pendant 24 heures dans différents solvants, tels que l'eau, l'éthanol ou l'acétate d'éthyle $(5 \mathrm{~g} / \mathrm{I} 0 \mathrm{ml})$. Certains extraits aqueux ont été autoclavés afin de tester la persistance du pouvoir inhibiteur à la chaleur. 


\section{4. - Tests microbiologiques}

I. 4.I. - Méthodes des antibiogrammes.

Les méthodes, aujourd'hui classiques, utilisées pour l'étude des antibiotiques ont été employées afin de déterminer qualitativement les propriétés inhibitrices vis-à-vis des différentes souches bactériennes des feuilles ou des aiguilles. Parmi ces méthodes, nous avons choisi les tests de diffusion et, en particulier, la méthode des antibiogrammes dont la technique est la suivante :

- disques de papier imprégnés d'extraits foliaires : on utilise du papier buvard découpé en rondelles de $5 \mathrm{~mm}$ de diamètre. Les disques, après stérilisation à l'autoclave sont plongés un par un dans les différents extraits. Les disques imprégnés de substances inhibitrices sont placés sur une plaque de verre stérile et mis à l'étuve pour le séchage $\left(37^{\circ} \mathrm{C}\right)$.

- préparation des boîtes et ensemencement : on coule en boîte de Petri $25 \mathrm{ml}$ de bouillon nutritif gélosé. Les boîtes sont séchées 30 minutes à $37^{\circ} \mathrm{C}$ avant l'emploi. L'inoculum, constitué d'une culture en phase exponentielle, doit être dilué de façon à obtenir sur la gélose des colonies denses mais non complètement confluentes. Après ensemencement, on pose les disques en appuyant légèrement.

- lecture des résultats : la lecture s'effectue en mesurant les zones d'inhibition autour de chaque disque. Nous avons classé les différentes zones d'inhibition selon l'échelle suivante :

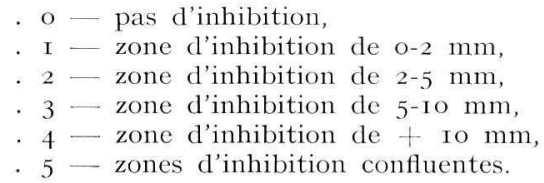

Par la méthode des antibiogrammes décrite ci-dessus, nous avons testé le pouvoir inhibiteur éventuel de chaque solvant utilisé lors de la lixiviation des feuilles broyées.

I. 4.2. - Méthode d'étude des phytoncides volatils.

Une autre technique a été mise au point afin d'estimer plus directement l'activité phytoncide des substances émises par le feuillage préalablement broyé au Waring-Blendor. A près préparation et ensemencement des boîtes de Petri selon la méthode décrite ci-dessus, on dispose sur le couvercle de chaque boîte 2 ou $5 \mathrm{~g}$ de feuilles fraîches broyées, puis on ferme chaque boîte à l'aide de parafilm (fig. I).

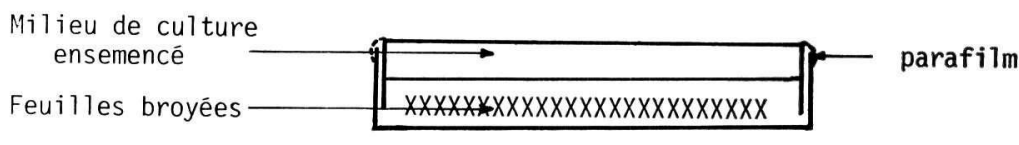

IFIG. I. - Dispositif utilisé afin de mesurer l'activité phytoncide de feuilles broyées

FIG. I. - Device used to measure the phytoncid activity of pulverized leaves

Simultanément, on prépare une échelle de lecture de référence en ensemençant des boîtes de Petri avec l'inoculum dilué au I/IO, au I/IOo, au I/I ooo et au I/Io ooo.

- lecture et interprétation des résultats : chaque boîte où s'est effectuée la croissance des survivants aux phytoncides volatils est comparée au point de vue de la densité des colonies aux boîtes de l'échelle. Si la densité des colonies est égale à celle obtenue avec :

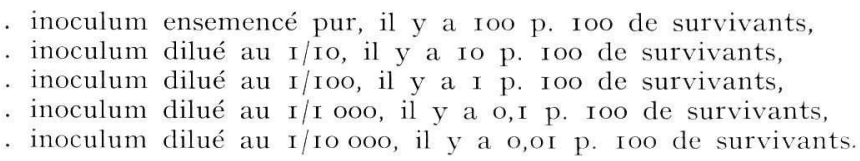




\section{II. - RÉSULTATS}

Les premières séries d'expériences effectuées montrent la spécificité des antibiotiques produits ou émis par les feuilles broyées. Nous analyserons successivement plusieurs facteurs :

- spécificité à l'égard des souches bactériennes,

- variation de l'activité phytoncide en fonction des différentes essences testées

- variation de l'activité phytoncide en fonction des méthodes employées.

\section{I. - Spécificité à l'égard des souches bactériennes}

Au cours de nos essais, de toutes les souches bactériennes testées, seule Escherichia coli s'est montrée résistante aux principes actifs contenus ou émis par les feuilles des différentes essences testées. Il importe cependant de signaler que les travaux de FEREnCZy et al. (I972) ont montré la sensibilité d'Escherichia coli à l'action inhibitrice des aiguilles de Picea abies et des feuilles de Populus marylandica, Carpinus betulus, Alnus rubra et Acer saccharinum.

Les autres souches bactériennes sont sensibles à des degrés divers aux substances inhibitrices contenues dans les feuilles (fig. 2 à 4). Bacillus cereus (fig. 3), ainsi que Staphylococcus aureus (fig. 2) sont parmi les espèces bactériennes testées les plus sensibles. Il est à noter que cette dernière espèce est couramment employée dans les études de sensibilité aux antibiotiques comme souche de référence.

\section{2. - Variation de l'activité antibiotique en fonction des différentes essences testées}

Bacillus cereus est la souche bactérienne la plus sensible à l'ensemble des essences forestières testées. Cependant, des différences notables dans la réponse aux tests microbiologiques sont enregistrées en fonction des essences utilisées (fig. 5 à 7 ).

La technique des antibiogrammes (disques imprégnés d'extraits alcooliques) permet de classer les différentes espèces de feuillus et de résineux en fonction de leurs propriétés inhibitrices vis-à-vis des trois espèces bactériennes sensibles :

- zones d'inhibition classe 5 : Chamacyparis mutkaensis

classe 4-3: Abies alba, Sorbus aucuparia

classe 3-2 : Pimus silvestris, Populus trichocarpa, Larix decidua

classe 2-I : Cedrus atlantica

\section{3. - Variation de l'activité antibiotique en fonction des techniques employées}

Au cours de cette étude, des variations importantes de l'activité antibiotique ont été observées en fonction, d'une part des méthodes d'extraction et, d'autre part, des tests microbiologiques employés. 

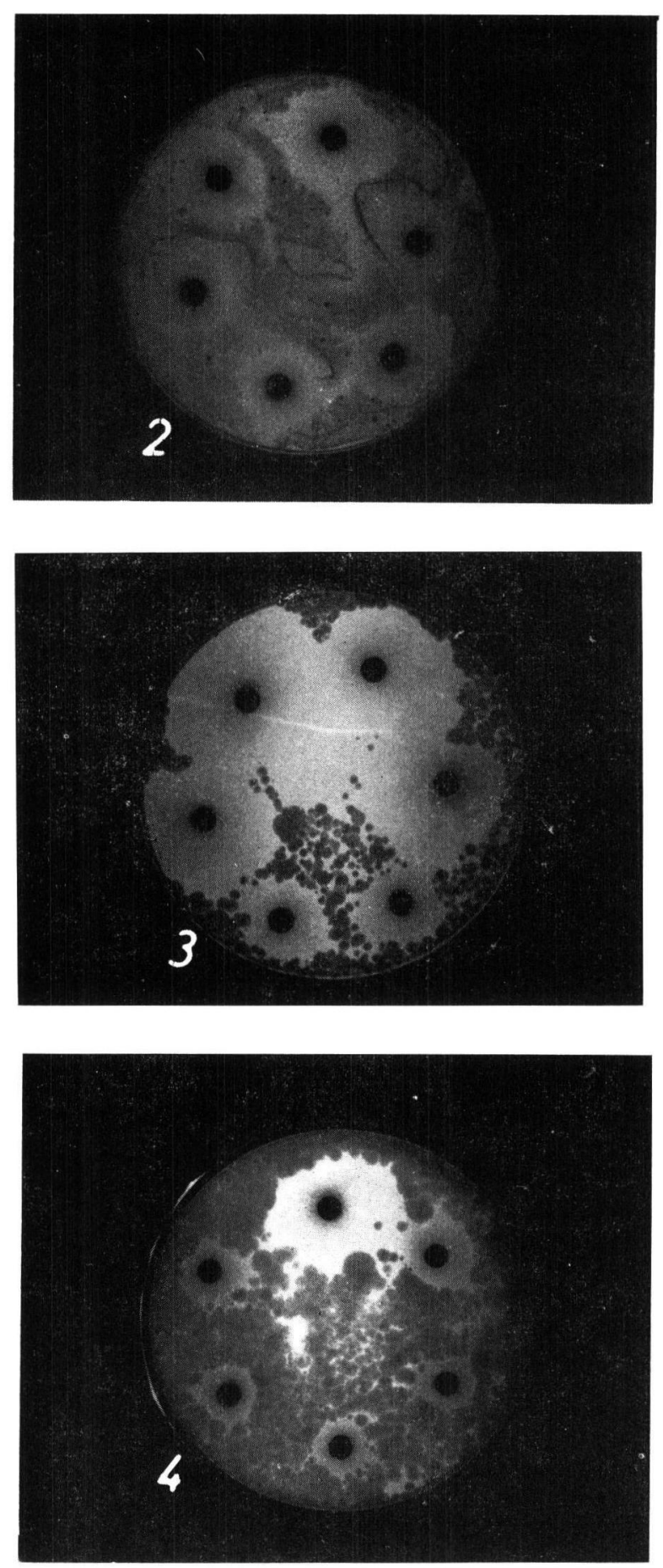

FIG. 2-4. - Spécificité de l'extrait alcoolique de Chamaecyparis nutkaensis à l'égard de souches bactériennes :

Staphylococcus aureus (fig. 2), Bacillus cereus (fig. 3), Bacillus thuringiensis (fig. 4)

Specificity of the alcohol extract from Ch. nutkaensis regarding bacterial strains : S. aureus (fig. 2), B. cereus (fig. 3), B. thuringiensis (fig. 4) 

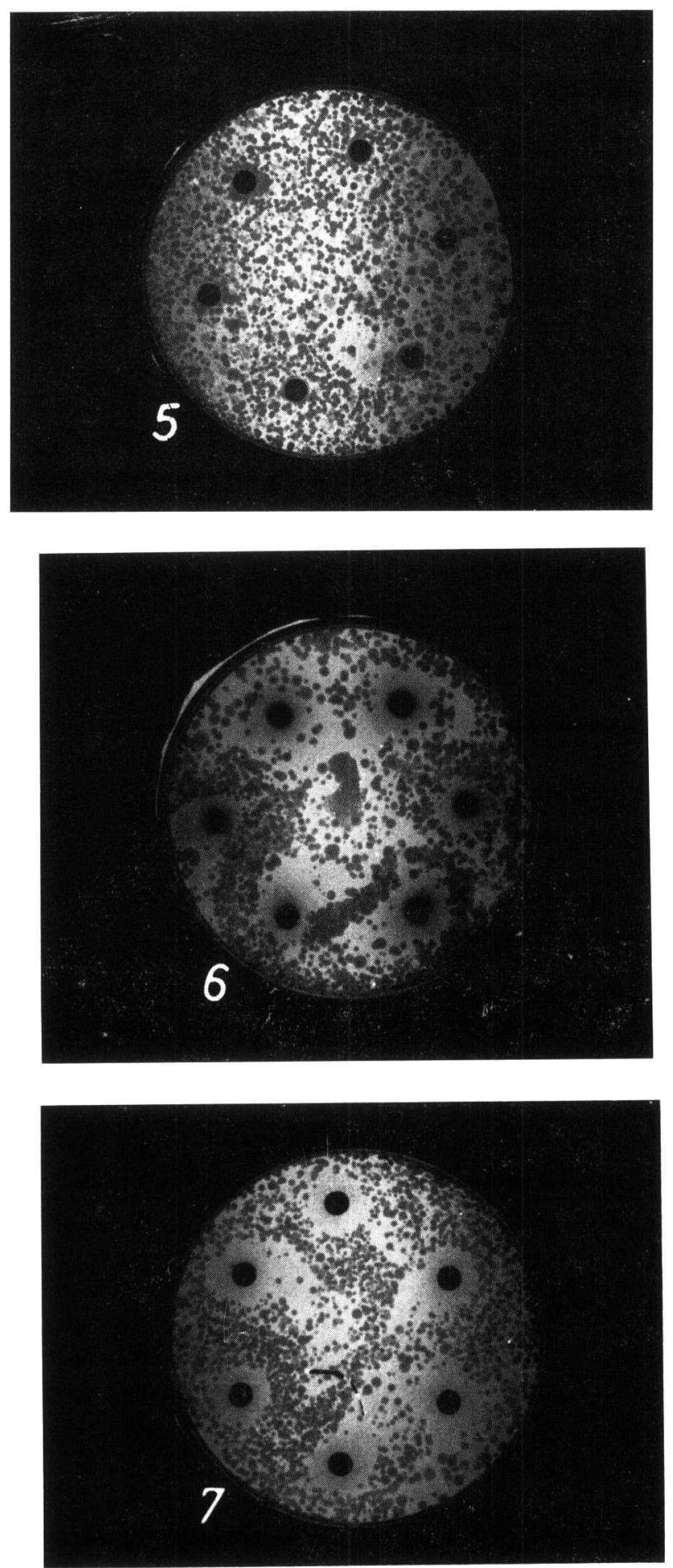

Fir. 5-7. - Variation de la sensibilité de Bacillus cereus vis-à-vis de différents extraits alcooliques:

Témoin (fig. 5), Thuya plicata (fig. 6), Abies alba (fir. 7)

Variations of the sensitivity of B. cereus toward various alcolol extracts : control (fig. 5), T. plicata (fig. 6), A. alba (fig. 7) 


\section{Méthodes d'extraction.}

Selon les solvants employés, on observe une nette différence de l'activité antibactérienne d'une même espèce d'arbres vis-à-vis d'une souche donnée. C'est ainsi que les extraits alcooliques de Thuya plicata, prélevés au mois de janvier sont très actifs vis-à-vis des bactéries testées, tandis que les extraits effectués avec de l'acétate d'éthyle sont beaucoup moins actifs (tabl. I) :

\section{TABLEAU I}

Variation de l'activité inhibitrice de Thuya plicata en fonction des solvants employés

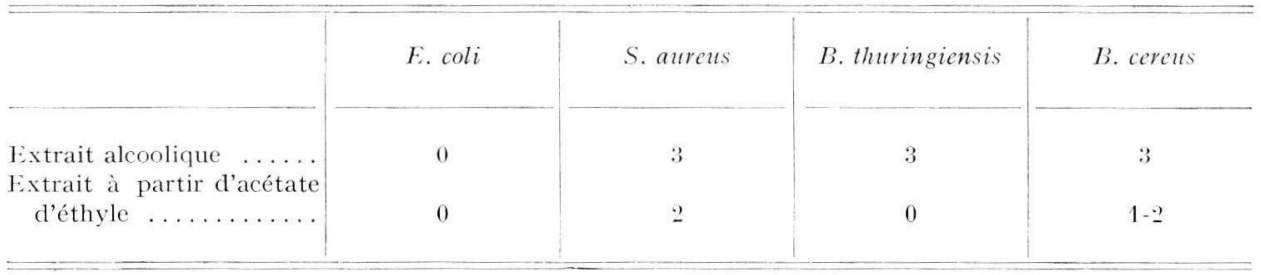

De plus, l'extrait aqueux de Chamaecyparis nutkaensis est le seul à conserver ses propriétés inhibitrices après passage à l'autoclave ( $1^{\circ} 0^{\circ} \mathrm{C}$ pendant $20 \mathrm{mn}$ ).

\section{Tests microbiologiques.}

Des réponses différentes peuvent en outre être observées selon les tests microbiologiques employés.

Des feuilles de Thuya plicata prélevées au printemps furent séparées en 2 lots après broyage. L,e premier servit à effectuer des extraits alcooliques qui se sont révélés inactifs à l'égard des bactéries Gram+ testées. Par contre, par la méthode des phytoncides volatils, $5 \mathrm{~g}$ de feuilles du deuxième lot ont inhibé 99,9 $\mathrm{p}$. Ioo de la population bactérienne ensemencée (Staphylococcus aureus)

\section{III. - DISCUSSION E'T CONCLUSION}

Cette étude confirme l'universalité des substances antibiotiques présentes dans le règne végétal et, en particulier, chez les essences forestières.

Cependant, si la mise en évidence de phénomènes inhibiteurs est relativement facile, les méthodes utilisées ne permettent pas de déceler l'existence de substances phytoncides au sein d'un peuplement forestier. L'utilisation, dans les différents tests, de feuilles broyées entraîne en effet des modifications chimiques importantes, telles que les phénomènes d'oxydation (oxydation des composés phénoliques par exemple).

De plus, les méthodes employées ne permettent pas de déterminer avec certitude si dans les conditions naturelles une espèce forestière est biologiquement plus active qu'une autre : par exemple, la méthode des antibiogrammes a uniquement 
été utilisée dans cette série d'expériences, afin de mettre en évidence les substances inhibitrices contenues dans les différents feuillages, mais nous ignorons totalement la charge initiale des disques en substances antibiotiques qui seule assurerait la fiabilité d'une telle méthode et permettrait de comparer les résultats obtenus à partir des différentes essences testées.

Seule la détermination de la concentration minimale inhibitrice de chaque extrait permettra de quantifier in vitro l'activité antibiotique des différentes essences forestières.

La détermination des différents composés chimiques possédant des propriétés inhibitrices pourra être envisagée ultérieurement.

Très peu de travaux ont été effectués directement sur le terrain et nous ne disposons pas encore des éléments indispensables qui caractériseraient les propriétés phytoncides des forêts et des principales essences forestières. Nous envisageons donc de mettre en évidence 1'existence de substances volatiles antibiotiques émises par certaines essences forestières en enceinte d'exposition dans une première étape, puis in situ.

Reçu pour publication in mars $19 \% 6$.

\author{
SUMMARY
}

PRELIMINARY NOTE ON THE PRESENCE

OI ANTIBIOTIC COMPONENTS IN FOREST TREES

This preliminary study gives some evidence of an antimicrobial activity in the foliage of several broad leaved and coniferous forest trees. Several microbiological tests have been undertaken in the laboratory : antibiograms and study of the volatile let off by pulverized leaves.

Several factors were analysed : specificity regarding bacterial strains, variability of phytoncidal activity in relation to the various studied species on the one hand, and to the different methods on the other hand.

\title{
RÉFÉRENCES BIBLIOGRAPHIQUES
}

Aclin-Erdman G, r950. Studies in tropolone series. 1. Thuyaplicins and nootkatin. Acta Chem. Scand., 4, IO3I-IO4I.

Erdtman H., Gripenberg S., I948. Antibiotic substances from the heartwood of Thuya plicata D. II. The constitution of $\gamma$ thuyaplicin. Acta Chem. Scand., 2, 625-638.

lierenczy L., Zsolt J., Laxtos S., I972. Screening for antibacterial, antifungal and antiphage substances in higher plants. Acta bio!., 18 ( I-4), 93-I I6.

HubBes M., I966. Inhibition of Hypoxylon pruinxtum (KLor/scme) CKE by aspen bark meal and the nature of active extractives. Can. J. Bot., 4, 365-386.

Hubbes M., Jung S., I973. Untersuchungen über die jahreszeitliche Hemmenwirkung von Eschenrindenextrakten auf das Wachsturm von Bacillus cereus. Phytopathol. Z., yy, 247-277.

Jung J., I965. Untersuchungen über Bakterien und Pilzhemmstoffe in höheren Pflanzen. Fortwiss. Cbl., $84(3-4)$ 96-I 24.

Klopping H. L., Van der Kerr G. J. M., 195I. Antifungal agents from the bark of Populus candidans. Nature, $\mathbf{1 6 \%}, 996$.

Michel M. F., i975. Les phytoncides. Ftude bibliographique. Station de Recherches sur la Forèt et l'Environnement Doc. 75-1 I, 46 p. 
Peterkova I., Peterka V., I972. Saisonbedingte Veränderungen der phytonziden Aktivität von Geholzen in Beziehung zu meteorologischen Faktoren. Acta Fac. Rerum nat. Univ. Comenianae Physiol. Plantarum, 5, 85-84.

Smirnoff W. R., I967. Effet des substances volatiles émises par le feuillage des plantes sur la survivance de 6 variétés du groupe Bacillus ceveus. Phytoprotection, 48 (3) I I9-127.

Smirnoff W. R., I968. Effects of volatile substances released by foliage of various plants on the entomopathogenic Bacillus cereus group. J. Invert. Pathol., 81 (3), 5 I $3-5$ I5.

Smirvoff W. R., I972. Effects of volatile substances released by foliage of Abies balsamea. J. Invert. Pathol., 19, 32-35.

Tokin B. P., I929. In Les phytoncides, B. P. Tokın. Académie des Sciences de 1'U.R.S.S., Moscou, I946.

Vullemin P., r889. In Antibiotics Historical introduction, p. 525. H. Florey. Oxford Univ. Press, London, New York, Toronto, I 949. 